\title{
The Experiment and Simulation of Propagation of Elastic Waves in Functionally Graded Materials with Defects
}

\author{
Dan Xue ${ }^{*}$, Qiwen Liu ${ }^{2}$, Guoqiang Luo ${ }^{3}$, Qiang Shen ${ }^{3}$, Lisheng $\mathrm{Liu}^{2}$, Dongfeng Cao ${ }^{4}$
}
${ }^{1}$ Department of Engineering Structure and Mechanics, Wuhan University of Technology, Wuhan, 430070, P.R. China, danxue_whut@163.com (Corresponding Author)
${ }^{2}$ Department of Engineering Structure and Mechanics, Wuhan University of Technology, Wuhan, 430070, P.R. China, qiwen_liu@whut.edu.cn

${ }^{3}$ State Key Laboratory of Advanced Technology for Materials Synthesis and Processing, Wuhan, University of Technology, Wuhan 430070, P.R.China,luoguoqiang1980@sina.com

${ }^{4}$ State Key Laboratory of Materials Synthesis and Processing, Wuhan University of Technology, Wuhan, 430070,P.R.China, caodongf@163.com

\begin{abstract}
The experiment of ultrasonic nondestructive testing and the related simulations have been performed to investigate the quality of the functional graded material with defects. The acoustic impedance of this materials is changed gradually along the direction of thickness. Water immersion method is used to filter shear wave in experiment, where A scan and C scan are combined to find defects. The results indicate that the phase position of waveform at the interface is the same as the incident wave when the functional graded material is of no defect. Otherwise, the phase position of waveform is opposite from at the incident wave at defective field. And the reason of frequency changing in experiment is explained. Then transient numerical simulations of the model are performed in this material using the same loading with the experiment. The results of numerical simulations are used to explain the mechanism of the experiment, and should have important guiding significance for the experiment.
\end{abstract}

Keywords: Functionally graded material; Numerical simulation; Nondestructive; Immersion method

\section{Introduction}

Functionally Graded Materials (FGMs) are special functional materials, with the composition, structure and properties varying gradually along the direction of thickness, which is different from the conventional materials. Because of their varying properties, FGMs are widely used. However, FGMs are produced by welding generally, there are defects exist in materials possibly. Therefore ultrasonic nondestructive testing with a transducer in immersion is used to test the integrity of FGMs. Due to a plurality of materials constitute, it is difficult to detect whether defects exist. Consequently, many scientists have do research in this area. Wave propagation in a simple one-dimensional model of FGMs was analyzed to optimize their resistance to failure by H. A. Bruck in 2000 [2]. Elastodynamic wave propagation in FGMs was simulated and experimented by J. Vollmann et al in 2006 [3]. And in 2014 Marie-Aude Ploix et al measured the ultrasonic scattering attenuation in austenitic stainless steel welds [5]. Not only that, but FGM seminars also have been held every two years from 1990 continued up to now. But because of a small defect sometimes masked by the echo from a larger nearby reflector, and it is difficult to detect, particularly for ultrasonic inspection of layered structures [6], the FGMs' detection still need to be optimized. So it is important to learn the mechanism of elastic wave propagation in FGMs in defect detection, and the combination of numerical simulations and experiments in FGMs' detection has not been reported so far.

In this paper, in order to investigate ultrasonic field thoroughly [7], ultrasonic non-destructive testing with a transducer in immersion was used to develop the experiment of a two-dimensional model composited by three kinds of linear elastic, isotropic metals. And the reason of frequency changing was explained. Then a simulation for the same models were presented to explain the mechanism of the experiment. In order to forecast propagation of elastic waves in functionally graded materials 
with defects, simulations of materials with defects presented were subsequently. In this work, mechanics was applied in FGM's testing, which has important guiding significance for the experiment and is a cross-disciplinary research.

\section{Theory of ultrasonic nondestructive testing}

The propagation of elastic waves is produced by vibration of molecules in the material. The vibration transmitted from a molecule to adjacent molecules, which keep spreading to surroundings. In immersion ultrasonic testing, both the transducer generating the wave and the sample to be tested are immersed in the alcohol. Elastic waves are generated by a piezoelectric transducer, then spread and focus through the acoustic lens in the coupling medium. Elastic waves starts to be received with the reverse operation when generating ultrasonic, after the measured sample interacted with elastic waves. The transducer collect the reflected waves and transform them into a plane wave and reconverted it into electrical signals.

The acoustic source producted by transducer is composed of a lot of little acoustic sources, which radiate spherical waves to the surrounding area. According to the superposition principle and mathematical operation, the total sound pressure at a point in the sound field is given by[4],

$$
\mathbf{P}=\frac{P_{0} S}{\lambda x}\left[\frac{2 J_{1}(k a \sin \theta)}{(k a \sin \theta)}\right] e^{j(\omega t-k r)}
$$

where $P_{0}$ is sound pressure amplitude of the acoustic source, $k$ is wave number, $\lambda$ is wavelength, $a$ is radius of piezoelectric wafer, $J_{1}$ is known as First-order Bessel function, and $x$ is distance from the tested point to the acoustic source.

\section{Experimental and numerical model}

\section{1. experimental model}

Wave spreads from material with lower impedance to that with higher impedance, the phase position of waveform at the interface is the same as incident wave. Conversely, the phase position of that is reversed. In order to test whether defects exist in the materials, the acoustic impedance of this materials is changed gradually along the direction of thickness. If the sample has a crack, which is filled by air. The impedance of it is zero. The phase position is opposite in the crack compared with the waveform at the interface.

Considering the above acoustic properties, TC4/ OFC/ 93W alloys welded to be tested are shown in Fig. 1. The thickness of the three layers are $0.2974 \mathrm{~mm}, 0.3186 \mathrm{~mm}$ and $4.894 \mathrm{~mm}$. Materials' parameters are given in Table. 1. Frequency of the waves produced by the transducer is $100 \mathrm{MHz}$, and the coupling medium between piezoelectric transducer and sample is alcohol concentration of $95 \%$.

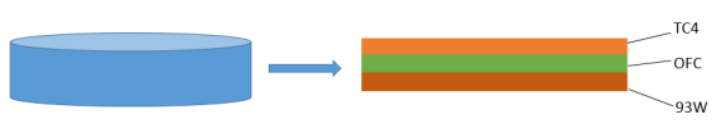

Fig. 1. Experimental model

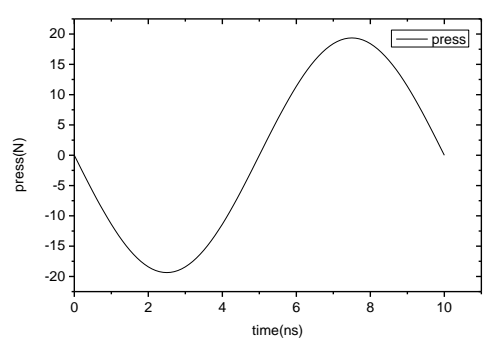

Fig. 2. The impulsive loading

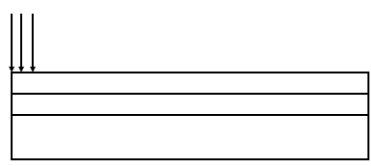

Fig. 3. The numerical model

Table 1. Material Parameters

\begin{tabular}{llll}
\hline Material & Young's modulus $(\mathrm{GPa})$ & Poisson's ratio & Density $\left(\mathrm{kg} / \mathrm{m}^{3}\right)$ \\
\hline TC4 & 118.7 & 0.33 & 4430 \\
OFC & 117.2 & 0.329 & 8940 \\
$93 \mathrm{~W}$ & 393 & 0.248 & 17640 \\
\hline
\end{tabular}

\subsection{Numerical model.}

Considering the shape of the sample, axisymmetric models were used in numerical simulations. According to the Eq. (1), the load at the surface of sample is obtained and shown in Fig. 2. Because focal length is artificial adjusted and imprecise, the impulsive loading is applied at a very small region of the plate upper surface, which shown in Fig. 3. The materials in the sample is assumed with no fusion of the metal layer. 


\section{Results}

\subsection{Results without defects}

W-Cu-Ti system FGM without defects was tested in immersion, and the result of A-scan is shown in Fig.4. The propagation path of the reflected waves starts from the position where time is about 10210ns. From the picture, waves reflected by interface are illegible. The frequency domain is much wider than the loading frequency domain, because that the high-frequency waves were absorbed by the coupling medium (alcohol) in the propagation process [1] following the Eq. (2):

$$
\mathrm{I}=I_{0} e^{-\beta y}
$$

where, $y$ denotes the distance waves spreading, $I_{0}$ represents the sound intensity at $y=0$, and the absorption coefficient of the sound energy $\beta$ is given by:

$$
\beta=a \times 10^{-11} f^{2}
$$

where $a$ is bigger than 4 (when in the air a=4), $f$ is the frequency of the initial frequency.

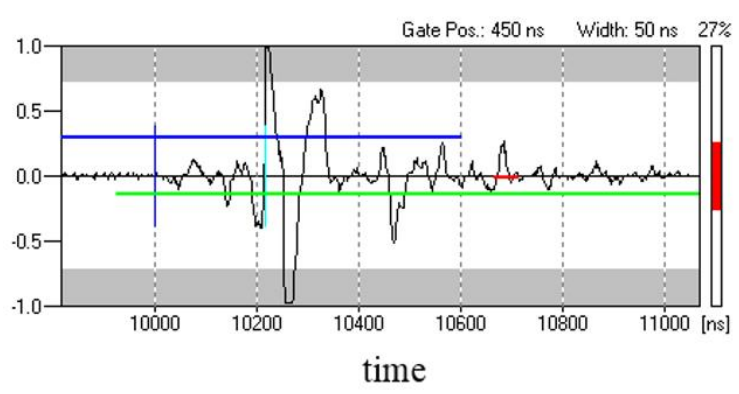

Fig. 4. The curve of A-scan experimental result

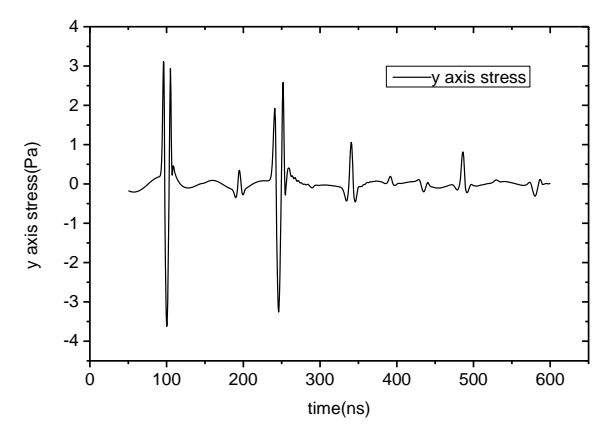

Fig. 5. The curve of numerical result without defects

Then we consider the stress wave propagation in $\mathrm{W}-\mathrm{Cu}-\mathrm{Ti}$ system FGM without defect using the same loading with the experiment. The result of numerical simulation is shown in Fig. 5, where waves reflected by the top surface is neglected. According to the relationship between time and velocity, the time of wave reflected by the first interface and the second face can be obtained as follows:

$$
\mathrm{t}_{i}=\frac{2 h_{i}}{c_{i}} \quad(i=1,2,3)
$$

where $h_{i}$ is the thickness of the $i$ layer, and $c_{i}$ is the longitudinal wave velocity of the $i$ layer. In Fig. 5 we can clearly get two distinct waves reflected at the interfaces. One is at about $100 \mathrm{~ns}$, the other one is at about 240ns. Since the materials are made by alloys, the material parameters is not very precise. And because a large number of high-frequency waves to be absorbed, there are some differences between experiment and numerical simulation without considering alcohol. However, the method of judging whether defects exist in the sample is the same.

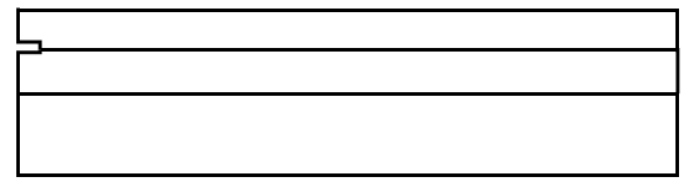

Fig. 6. The numerical model with a defect in the first interface

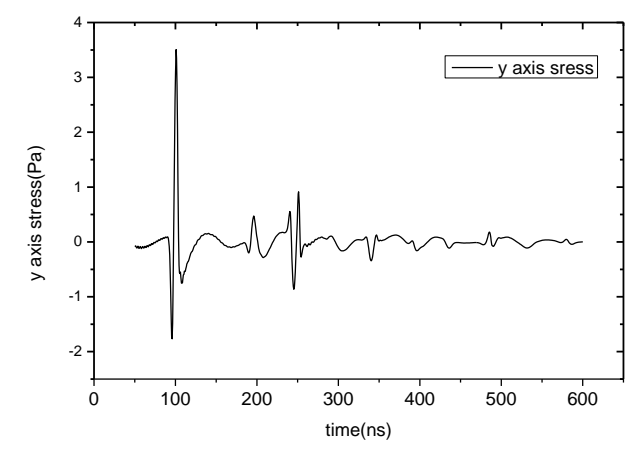

Fig. 7. The curve of numerical result with a defect in the first interface

\subsection{Results with defects}

We turn to W-Cu-Ti system FGM with defects problem. We put this problem into two sections. In the first section, the sample has a defect in the first interface, as shown in Fig. 6, and the defect is so small that it can be considered as a welding seam. The same strategy was applied for numerical model with defects. Apparently in the result we can see there are changes in the first crest that the stress curve line reach peak turning into going down to reach peak then go up, as shown in Fig. 7. In other words, the phase position of waveform at the interface turned over. At the same time, the wave reflected by the second 
interface is illegible, because of the defect in the first interface affecting wave propagation.

In the second section, we assume that the defect exists in the second interface as illustrated in Fig. 8. And the result of numerical simulation obtained is shown in Fig. 9. From the result, the phase position of crest at the first interface remained still, but the crest at the second interface became to a small crest. The small crest represents the superposition of reflection on the top face of the defect and the second reflection of the first interface.

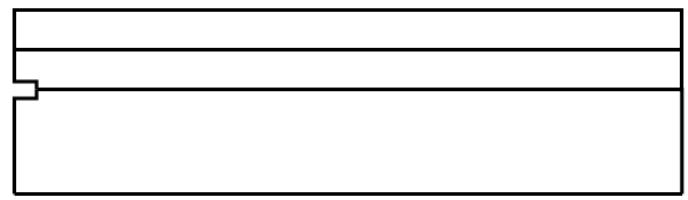

Fig. 8. The numerical model with a defect in the second interface

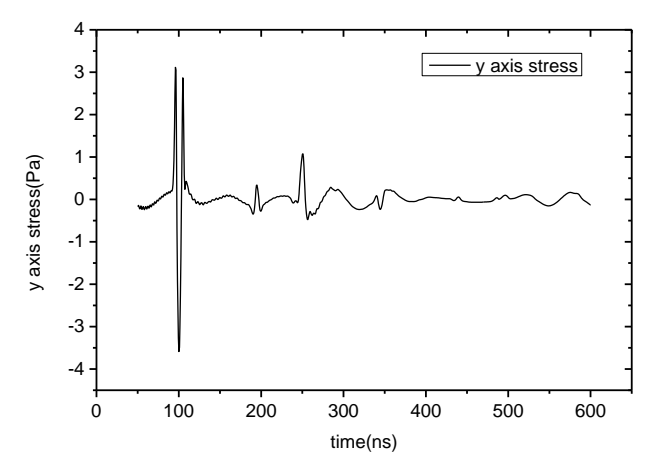

Fig. 9. The curve of numerical result with a defect in the second interface

\section{Conclusions}

In this paper, experiment and numerical simulations were used to compare wave propagation in W-Cu-Ti system FGMs and explain the reflected waves on the interface. In the two numerical simulations, defects were in different locations, one in the first interface and the other in the second interface. The experimental and numerical results about the sample without defects show a significant difference. The main reason of the difference is the coupling medium absorbing high-frequency waves. And the illegible experimental result suggests that we couldn't find the interface if we only use A-scan, so A-scan and C-scan which is used to record the intensity of the reflected wave and convert to pixels with gray scale signal must be combined to test in fact. While on the basis of the experiment, numerical simulations were simplified without fusion of the metal layer. The results are in good agreement with theory, and crests at the interface is clearly.

Actually, preparation FGMs exits defects likely, so numerical simulations with a defect developed subsequently. Compared two pictures (Fig. 5 and Fig. 7), the shape of the wave has changed at the first crest in Fig.6, according this we can judge the existence of a defect in the first interface. Then compared Fig. 5 and Fig. 9, the first crests in these two picture are the same, the phase position of waveform without change in Fig. 9, so there is no defects in the first interface. But the second crest changed, which is original big crest turning into a small one without phase position changed, because of superposition of the second reflected waves in the first interface and the reflected waves in the second interface exists affection.

It should be noted that the constitution of FGMs, the existence of the error resulted by artificial operation, and the welding quality of the FGMs may affect the experimental results. And attenuation of the specific size of high-frequency waves in immersion ultrasonic testing and wave velocity of FGMs are not known yet. Nevertheless, at least the principle of wave propagation can be explained by numerical simulations. Moreover we can determine whether defects exist and which layer defects in through comparing stress wave propagation pictures without defects and defects existence, which has significant for the future research about wave propagation in complicated FGMs, and we can make a map of ultrasonic testing to help artificial operational testing. Further studies are in progress.

\section{Acknowledgements}

This work has been supported by National Natural Science Foundation of China ( 51202175 ) and the 111 Project ( B13035 ) and the Fundamental Research Funds for the Central Universities ( WUT:2013-II-024 ) and State Key Laboratory of Advanced Technology for Materials Synthesis and Processing(Wuhan University of Technology)(Grant No: 2014-KF-7).

\section{References}

1. Feng Shu, The effect of the speed sound caused by sound absorption. Engineering course physics 2(1993) 8-9.

2. H. A. Bruck, A one-dimensional model for designing functionally graded materials to manage stress wave. International Journal of Solids and Structures.37 (2000) 6383-6395.

3. J. Vollmann et al, Elastodynamic wave propagation in graded materials: simulations experiments, phenomena, and applications. Ultrasonics 44 (2006) 1215-1221.

4.Junzhe Zhang, Nondestructive testing technology and its application, Science Press, Beijing, 2010.

5. M.-A. Ploix et al., Measurement of ultrasonic scattering attenuation in austenitic stainless steel welds: Realistic input data for NDT numerical modeling, Ultrasonics (2014), http://dx.doi.org/10.1016/j.ultras.2014.04.005.

6. G.-M. Zhang et al, Sparse signal representation and its applications in ultrasonic NDE. Ultrasonics 52 (2012) $351-363$.

7. X. Jian et al, A model for transient ultrasonic field in solid generated by a transducer in immersion. Sensors and Actuators A 133 (2007) $439-446$. 